\title{
Gene Expression of CD70 and CD27 Is Increased in Alopecia Areata Lesions and Associated with Disease Severity and Activity
}

\author{
Radwa El- Sayed Mahmoud Marie $\stackrel{D}{1},{ }^{1}$ Noha M. Abd El-Fadeel ${ }^{D},{ }^{2,3}$ \\ Yara El-Sayed Marei $\left(\mathbb{D},{ }^{4}\right.$ and Lina M. Atef $\mathbb{D}^{1}$ \\ ${ }^{1}$ Department of Dermatology, Venereology and Andrology, Faculty of Medicine, Suez Canal University, Ismailia, Egypt \\ ${ }^{2}$ Department of Medical Biochemistry and Molecular Biology, Faculty of Medicine, Suez Canal University, Ismailia, Egypt \\ ${ }^{3}$ Oncology Diagnostic Unit, Faculty of Medicine, Suez Canal University, Ismailia, Egypt \\ ${ }^{4}$ Department of Medical Microbiology and Immunology, Faculty of Medicine, Suez Canal University, Ismailia, Egypt
}

Correspondence should be addressed to Radwa El- Sayed Mahmoud Marie; rivercruise84@gmail.com

Received 1 November 2021; Accepted 15 February 2022; Published 8 March 2022

Academic Editor: Mohamed Elsaie

Copyright ( 92022 Radwa El- Sayed Mahmoud Marie et al. This is an open access article distributed under the Creative Commons Attribution License, which permits unrestricted use, distribution, and reproduction in any medium, provided the original work is properly cited.

\begin{abstract}
Background. Alopecia areata (AA) is an acquired hair loss disorder induced by a cell-mediated autoimmune attack against anagen hair follicles. CD27-CD70 is a receptor-ligand complex which enhances T helper and cytotoxic T cell activation, survival, and proliferation. The overstimulation of this complex can lead to a lack of tolerance and the development of autoimmunity. Objectives. This study aimed to assess the gene expression of CD27 and CD70 in patients with AA. Methods. CD70 and CD27 mRNA expressions were evaluated by a quantitative real-time polymerase chain reaction in scalp biopsies from 40 AA patients (both AA lesions and non-lesional areas) and 40 healthy controls (HCs). The Severity of Alopecia Tool (SALT) score was used to assess AA severity. Patients were evaluated for signs of AA activity, including a positive hair pull test and dermoscopic features of black dots, broken hairs, and tapering hairs. Results. The gene expression of CD70 and CD27 was significantly higher in AA lesions than in non-lesional areas $(p<0.001$ for both) and HCs $(p=0.004, p=0.014$, respectively). There were significant positive correlations between AA severity and gene expression of CD70 $(p<0.001)$ and CD27 $(p=0.030)$ in AA lesions. Significant associations were detected between signs of AA activity and lesional gene expression of CD70 and CD27. Additionally, CD70 and CD27 gene expression was significantly lower in non-lesional biopsies compared to HCs $(p<0.001)$. Conclusion. Gene expression of CD70 and CD27 was increased in AA lesions and was associated with disease severity and activity. Thus, both molecules can be a predictor of AA severity and activity. Furthermore, the expression was reduced in non-lesional scalp areas. Thus, a lack of CD27 and CD70 expression may initially predispose to immunological dysregulation and the development of AA.
\end{abstract}

\section{Introduction}

Alopecia areata (AA) is an acquired non-cicatricial hair loss disorder affecting $0.1 \%-0.2 \%$ of individuals worldwide [1]. A collapse of the hair follicle (HF) immune privilege causing a CD8+ cytotoxic T (cT) cell assault against anagen HFs is implicated in AA development [2,3]. However, the precise molecular mechanisms are still poorly established.

Cluster differentiation (CD) 27 is a member of the tumor necrosis factor receptor family. CD27 is expressed on natural killer cells, T helper (Th) cells, cT cells, and hematopoietic stem cells [4]. CD70, the uniquely identified ligand of CD27, belongs to the tumor necrosis factor family and is expressed exclusively on activated T cells, B cells, natural killer cells, and dendritic cells. The expression of CD70 is triggered by $\mathrm{T}$ cell activation upon antigen receptor attachment, toll-like receptor, or CD40 signaling. The interaction between CD70 on stimulated antigen presenting cells (APCs) and CD27 on $\mathrm{T}$ cells generates costimulatory signals that enhance $\mathrm{Th}$ and cT cell activation, survival, proliferation, chemotaxis, and 
cytokine production such as interleukin 2 and interferon- $\gamma$ [5]. Moreover, CD27-CD70 interaction induces the production of CXCL10 chemokine by activated cT cells, which enhances the chemotaxis of additional activated cT cells [6]. CD70 was also found to stimulate Th1 cell differentiation independent of interleukin 12 [7]. Soluble CD27 can be shed from the activated T cells' surface via cleavage by matrix metalloproteinases to be released in the circulation. It was reported that serum soluble CD27 could enhance T cells and antigen-primed B cells' activation and increase immunoglobulin $G$ production $[4,5]$. Interestingly, it was proposed that CD70 signaling generated by unaroused, premature APCs can inevitably lead to a lack of tolerance and autoimmunity development [8]. Significant evidence indicates that the dysregulation of CD27-CD70 complex signaling is involved in the pathogenesis of several immune-related disorders [5], but the pathway has been poorly investigated in AA. CD70 is merely expressed upon T cell activation, and thus blocking the C27-CD70 complex has been proposed as an appealing treatment target for autoimmune disorders [9]. This study aimed to assess the expression of CD27 and CD70 genes in patients with $\mathrm{AA}$ and to evaluate the association between this expression and AA severity and activity.

\section{Patients and Methods}

2.1. Participants. A total of 40 patients with AA were enrolled in this descriptive analytical case-control study. Patients were recruited from the Dermatology Outpatient Clinic, Suez Canal University Hospital, Ismailia, Egypt. The biochemical analyses were conducted at the molecular laboratory of the Oncology Diagnostic Unit, Faculty of Medicine, Suez Canal University, Egypt. The study was performed between February and September 2021, in line with the guidelines of the Helsinki Declaration and the items of the STROBE statement. Approval was granted by the Institutional Review Board and the Research Ethics Committee, Faculty of Medicine, Suez Canal University, on 25 January 2021, with the approval code: 4454. Age and sex-matched HCs with no concurrent infections, history of AA, autoimmune disorders, atopy, or cancer were included in the study. All participants signed written informed consent form.

Alopecia areata was diagnosed clinically through detecting patches of total hair loss with normal scalp skin, and the diagnosis was confirmed by dermoscopy examination. Exclusion criteria were patients who had any systemic treatment for three months or topical applications for 2 weeks prior to the study or had regrowth of hair, concurrent infection, or a history of other autoimmune diseases or atopy or cancer.

Patients' complete history was recorded including demographic data (age and sex) and significant clinical data (duration of the present AA lesions, disease course, age of disease onset, AA in other body sites, prior attacks of AA, family history of AA, atopy, or other immune-mediated disorders), and dermatological examination was performed to detect site of patches, their number and pattern, and presence of nail abnormalities. AA clinical pattern was categorized into patchy (single patch and multiple patches), ophiasis, alopecia totalis, and alopecia universalis. The "Severity of Alopecia Tool" (SALT) score [10] was used to evaluate AA severity. AA activity was assessed via the subjective history of progression, an objective examination of the hair pull test at the edges of each patch, and the presence of black dots, broken hairs, and tapering hair on dermoscopy examination (DermLite, 3 Gen LLC, San Juan Capistrano, CA, USA) (magnification $\times 10$ ).

2.2. Assessment of the Expression of CD70 and CD27 Genes. Punch scalp biopsies $(4 \mathrm{~mm})$ were taken from each patient from AA lesions and non-lesional areas. One scalp biopsy was taken from the patient with alopecia universalis and HCs. Biopsies were soon submerged in the RNA stabilizing solution (Qiagen, USA) and moved to $-80 \mathrm{C}$ for storage until handling. According to the manufacturer's instructions, we isolated the total RNA using RNeasy Mini Kit (QIAGEN, USA). We also assessed RNA purity by NanoDrop ND1000 spectrophotometer at the absorbance ratio of $260 / 280 \mathrm{~nm}$ (NanoDrop Tech., Inc., Wilmington, DE, USA). To assess the integrity of the RNA, we ran it at $1 \%$ agarose gel electrophoresis.

Quantitative real-time polymerase chain reactions (PCR) for CD27 and CD70 genes were performed on step one real-time PCR instrument (Applied Biosystems, UK) using COSMO cDNA synthesis kits (WF-1020500X, Willowfort, UK), HERA plus SYBR Green-qPCR Kit (WF1030800X, Willowfort, UK), and the specific primers for the target genes (Table 1).

The amplification program included two stages, an initial denaturation stage at $95 \mathrm{C}$ for $3 \mathrm{~min}$, followed by 40 cycles of $95 \mathrm{C}$ denaturation for $15 \mathrm{~s}$ and annealing at $57 \mathrm{C}$ for GAPDH and $60 \mathrm{C}$ for (CD27 or CD70) for $60 \mathrm{~s}$. After amplification, a melting curve analysis was performed to confirm PCR amplicons by collecting the fluorescence data. GAPDH was used as an internal control. The relative amounts of the target genes were calculated using the delta CT method.

2.3. Statistical Analysis. Statistical analysis was done via the Social Sciences Statistical Package. Categorical values were represented by using numbers and percentages. The normality of distribution was tested by the Kolmogorov-Smirnov test. Numerical values were represented using the range, median, mean, and standard deviation. Chisquare test, Monte Carlo correction, Mann-Whitney test, Kruskal-Wallis test, Wilcoxon signed rank test, and Spearman coefficient were used to measure the significance. The results were considered significant at a $p$ value less than 0.05 (confidence level of 5\%).

\section{Results}

3.1. Clinical and Demographic Data of AA Patients. In patients with AA, the age ranged from 15 to 62 years (mean age $=28.93 \pm 12.21$ years). Twenty $(50 \%)$ patients were males, and $20(50 \%)$ were females. In HCs, the age ranged from 18 to 59 (mean age $=29.80 \pm 10.16$ years). Eighteen (45\%) HC individuals were males, and 22 (55\%) were 
TABLE 1: Primers used for real-time polymerase reaction assay.

\begin{tabular}{lc}
\hline Gene & Sequence \\
\hline \multirow{2}{*}{ CD70 } & Forward: 5' - TTGGTGATCTGCCTCGTGGT-3' \\
& Reverse: 5' - CCTTGTCCAGCTCTGGTCCAT-3' \\
CD27 & Forward: 5' ACCCTCAGCCCACCCACTTA-3' \\
& Reverse: 5' - CAGGGTGAAAACAAGGAACATT-3' \\
& Forward: 5'-CGGAGTCAACGGATTTGGTCGTAT- \\
GAPDH & $3^{\prime}$ \\
& Reverse: 5'-AGCCTTCTCCATGGTGGT-3' \\
\hline
\end{tabular}

CD: cluster differentiation; GAPDH: human glyceraldehyde-3-phosphate dehydrogenase.

females. There was no statistically significant difference in age or gender between patients with AA and HCs (Supplementary Table 1). Clinical data of AA patients are shown in Table 2.

3.2. Expression of $C D 70$ and $C D 27$ Genes. The mean mRNA expression of the CD70 gene in AA lesions and non-lesional scalp skin was 2.41 fold and 0.16 fold, respectively, relative to the expression in HCs. The relative expression of CD70 gene was significantly higher in AA lesions compared to nonlesional areas $(p<0.001)$ and HCs $(p=0.004)$. In addition, the gene expression was significantly lower in non-lesional areas than in HCs $(p<0.001)$ (Figure 1).

The mean mRNA expression of the CD27 gene in AA lesions and non-lesional scalp skin was 3.19 fold and 0.25 fold, respectively, relative to the expression in HCs. The relative expression of the $\mathrm{CD} 27$ gene was significantly higher in AA lesions compared to non-lesional areas $(p<0.001)$ and HCs $(p=0.014)$. The CD27 gene expression was significantly lower in non-lesional biopsies compared to HCs $(p<0.001)$ (Figure 2).

The study revealed significant positive correlations between AA severity (SALT) and the relative mRNA expressions of CD70 $(p<0.001)$ (Figure 3(a)) and CD27 $(p=0.030)$ (Figure $3(\mathrm{~b}))$ in AA lesions.

Furthermore, there were significant associations between signs of AA activity (positive hair pull test, and the presence of black dots, broken hairs, and tapering hair on dermoscopy examination) and the relative gene expressions of CD70 and CD27 (Table 3). Apart from that, there was no significant relation between CD70 or CD27 gene expression and other data of AA patients (age, sex, disease course, age of onset, duration of AA lesions, clinical pattern, nail abnormalities or family history of AA, atopy, or immune-mediated disorders) (Supplementary Tables 2-5).

\section{Discussion}

In this study, the relative gene expression of CD70 and CD27 in AA lesions was significantly higher than that in nonlesional areas and HCs, correlated with AA severity, and was associated with signs of AA activity, including positive hair pull test at patch margins and dermoscopic features such as black dots, broken hairs, and tapering hair. AA is generally believed to be an autoimmune assault upon anagen $\mathrm{HF}$, orchestrated by Th1 and cT lymphocytes. Inflammatory
TABLE 2: Clinical data of patients with AA $(n=44)$.

\begin{tabular}{lcc}
\hline Clinical data & No. & $\%$ \\
\hline & Duration of AA lesions (months) & \\
<3 months & 8 & 20.0 \\
$3-12$ months & 11 & 27.5 \\
$12-24$ months & 5 & 12.5 \\
$2-5$ years & 9 & 22.5 \\
$\geq 5$ years & 7 & 17.5 \\
Min.-Max. & $0.25-168.0$ \\
Mean \pm SD & $26.98 \pm 39.75$ \\
Median (IQR) & $12.0(3.0-36.0)$
\end{tabular}

\begin{tabular}{|c|c|c|}
\hline \multicolumn{3}{|c|}{ Course } \\
\hline Progressive & 27 & 67.5 \\
\hline Fluctuating & 5 & 12.5 \\
\hline Stationary & 8 & 20.0 \\
\hline \multicolumn{3}{|c|}{ Age of disease onset (years) } \\
\hline Children $(0-<13)$ & 6 & 15.0 \\
\hline $\begin{array}{l}\text { Adolescents and young } \\
\text { youth }(13-<25)\end{array}$ & 16 & 40.0 \\
\hline Adulthood $(25-<40)$ & 10 & 25.0 \\
\hline Middle age $(40-<60)$ & 8 & 20.0 \\
\hline Min.-Max. & \multicolumn{2}{|c|}{$2.0-50.0$} \\
\hline Mean \pm SD & \multicolumn{2}{|c|}{$25.58 \pm 13.25$} \\
\hline Median (IQR) & \multicolumn{2}{|c|}{$\begin{array}{c}24.0 \\
(17.0-32.0)\end{array}$} \\
\hline $\begin{array}{l}\text { Alopecia in other sites } \\
\text { (beard or eyebrows) }\end{array}$ & 14 & 35.0 \\
\hline \multicolumn{3}{|c|}{ SALT score } \\
\hline S1 (<25\% hair loss) & 22 & 55.0 \\
\hline S2 (25-49\% hair loss) & 9 & 22.5 \\
\hline S3 (50-74\% hair loss) & 7 & 17.5 \\
\hline S4 (75-99\% hair loss) & 0 & 0.0 \\
\hline S5 (100\% hair loss) & 2 & 5.0 \\
\hline Min.-Max. & \multicolumn{2}{|c|}{$1.50-100.0$} \\
\hline Mean \pm SD & \multicolumn{2}{|c|}{$25.08 \pm 26.02$} \\
\hline Median (IQR) & \multicolumn{2}{|c|}{$12.0(3.0-42.0)$} \\
\hline
\end{tabular}

Patchy

Pattern

Single patch

Multiple separate patches

Multiple reticular patches

Ophiasis \pm other patterns

Ophiasis

Multiple separate patches and ophiasis

Ophiasis and sisaipho

Universalis

Previous attacks of AA

Family history of AA

Family history of atopy

Family history of autoimmune disorders

Nail changes (pitting, ridging, dystrophy)

Black dots

Broken hairs

Tapering hairs

$22 \quad 55.0$

$\begin{array}{ll}7 & 17.5\end{array}$

$12 \quad 30.0$

$3 \quad 7.5$

$16 \quad 40.0$

$8 \quad 20.0$

$7 \quad 17.5$

$1 \quad 2.5$

$2 \quad 5.0$

$22 \quad 55.0$

$3 \quad 7.5$

$\begin{array}{ll}5 & 12.5\end{array}$

$3 \quad 7.5$

$7 \quad 17.5$

$30 \quad 75.0$

$16 \quad 40.0$

SD: standard deviation; IQR: interquartile range. AA: alopecia areata; SALT : Severity of Alopecia Tool.

infiltrate of Th, cT cells, and APCs was identified in the peribulbar region of anagen HFs in active AA lesions and was found to be correlated with AA severity. These kinds of infiltrate trigger apoptosis in anagen HF keratinocytes, 


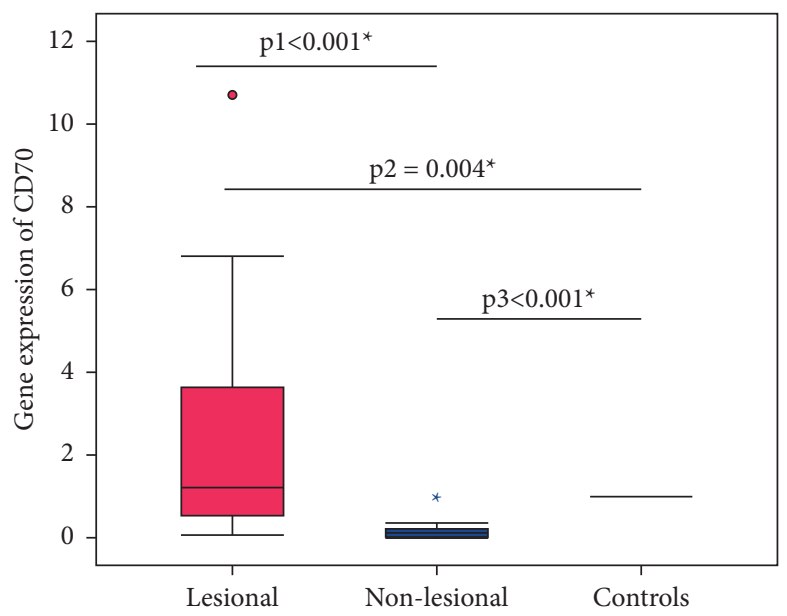

FIGURE 1: The relative expression of CD70 gene in patients with AA $(n=40)$ compared to HCs $(n=40)$. The relative CD70 gene expression in AA lesions ranged from 0.07 to 10.70 (mean $2.41 \pm 2.71$, median 1.22), while in non-lesional areas, it ranged from 0.01 to 0.82 (mean $0.16 \pm 0.19$, median 0.12). Wilcoxon signed rank test ( $\mathrm{p} 1)$ was used to compare the expression in AA lesions and non-lesional areas. Mann-Whitney test (p2) was used to compare the expression in AA lesions and HCs. Mann-Whitney test (p3) was used to compare the expression in non-lesional areas and HCs. ${ }^{*}$ Significant at $p<0.05$. AA: alopecia areata; HCs: healthy controls; CD: cluster differentiation.

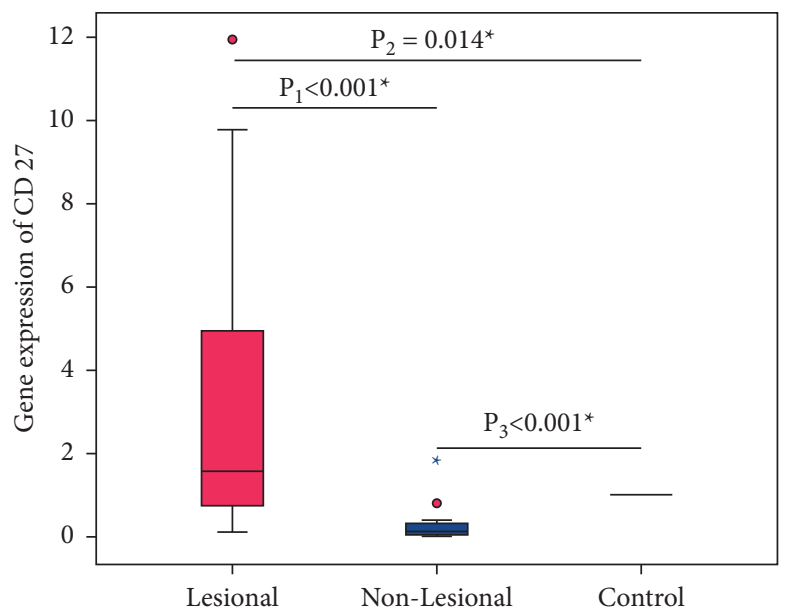

Figure 2: The relative expression of CD27 gene in patients with AA $(n=40)$ compared to HCs $(n=40)$. The relative CD27 gene expression in AA lesions ranged from 0.09 to 11.96 (mean 3.19 \pm 3.65 , median 1.58), while in non-lesional areas, it ranged from 0.0 to 1.68 (mean $0.25 \pm 0.38$, median 0.14). Wilcoxon signed rank test ( $\mathrm{p} 1)$ was used to compare the expression in AA lesions and non-lesional areas. Mann-Whitney test (p2) was used to compare the expression in AA lesions and HCs. Mann-Whitney test (p3) was used to compare the expression in non-lesional areas and HCs. ${ }^{*}$ Significant at $p<0.05$. AA: alopecia areata; HCs: healthy controls; CD: cluster differentiation.

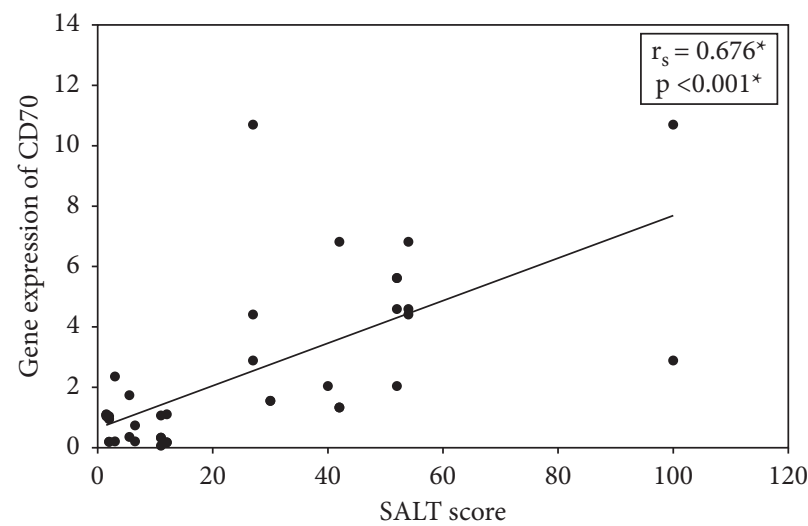

(a)

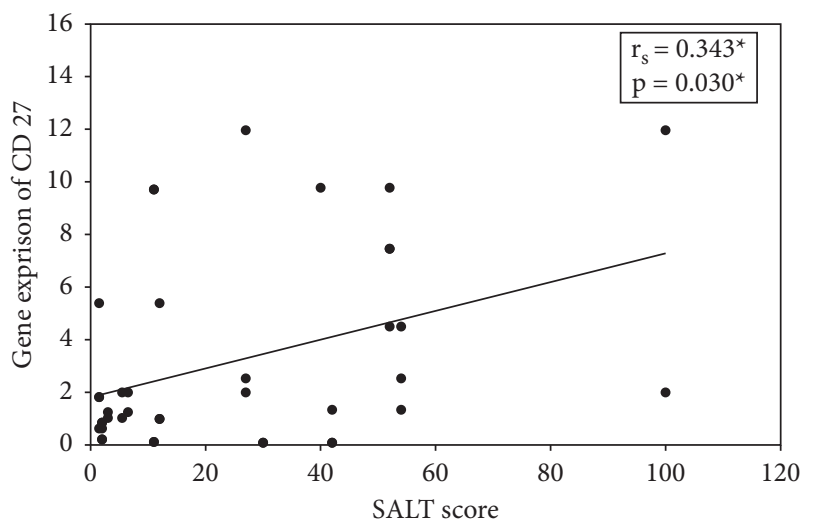

(b)

Figure 3: (a) The correlation between CD70 mRNA levels in AA lesions ( $n=40)$ and SALT score. (b) The correlation between CD27 mRNA levels in AA lesions $(n=40)$ and SALT score. rs: Spearman coefficient; ${ }^{*}$ significant at $p<0.05$; AA: alopecia areata; SALT: Severity of Alopecia Tool; CD: cluster differentiation. 


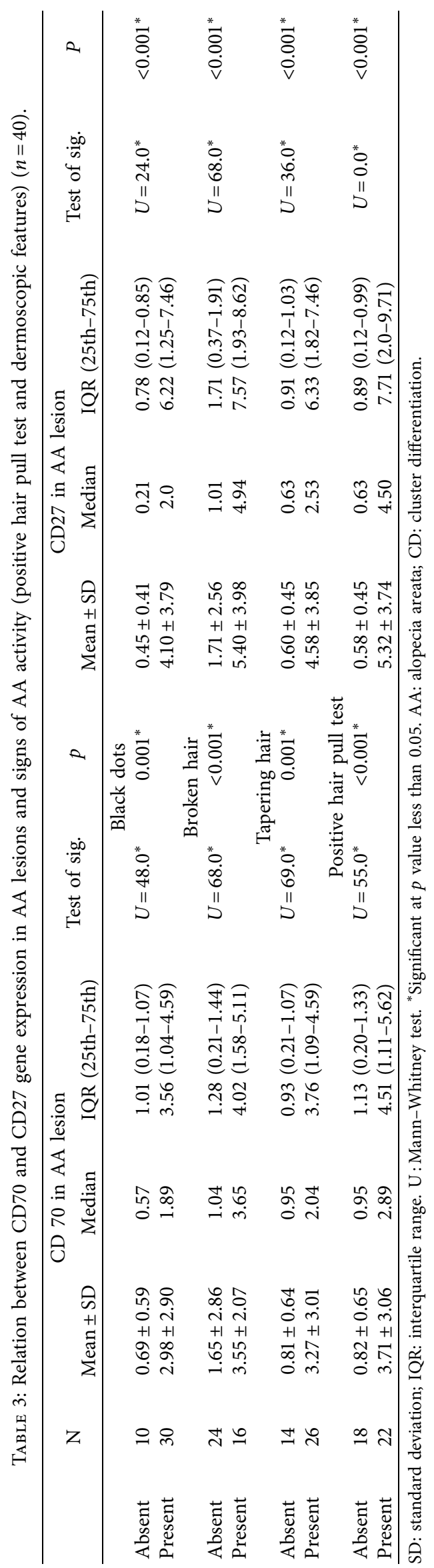


causing their arrest and regression into the telogen or dystrophic anagen states [2]. Clinically, this is manifested by a sudden stoppage of hair shaft growth, resulting in tapered and broken hair shafts with a positive hair pull test at the active patch margin [11]. The CD27-CD70 complex is mostly expressed on activated T cells, B cells, natural killer cells, and dendritic cells, and its interaction signaling facilitates Th1 and cT cell activation, survival, proliferation, and chemotaxis [5]. Thus, the higher gene expression of CD70 and CD27 in active AA lesions can be explained by the increased infiltration of immune cells expressing these molecules. This infiltration was present mostly in active AA lesions and was associated with AA severity; this fact may clarify the association between CD27 and CD70 gene expression and AA severity and signs of activity. As a result, CD27 and CD70 gene expression can be used to predict the severity and activity of AA. To date, no previous studies have evaluated the gene expression of CD27-CD70 complex in AA.

In agreement with our results, CD70 expression was upregulated on CD4+ Th cells isolated from the synovial fluid of patients with psoriatic arthritis [12]. CD70 expression was also increased in the synovium and peripheral blood mononuclear cells (PBMCs) of patients with rheumatoid arthritis [12, 13], systemic lupus erythematosus (SLE) [14], systemic sclerosis [15], and Sjogren's syndrome [16]. This expression correlated with the hypo or demethylation of the CD70 gene promoter area, which causes a failure to repress CD70 expression when it is triggered by T cell activation $[15,16]$. Regarding CD27, its soluble serum level was elevated in patients with active vitiligo and was suggested as a marker of disease progression $[17,18]$. Serum soluble CD27 was downregulated upon treatment of psoriasis [19]. CD27 expression was significantly elevated in the lesional skin and serum of patients with systemic sclerosis, with a significant association with disease severity [20]. The expression of CD27+ B cells and serum soluble CD27 was increased in SLE patients and correlated with disease activity [21]. The pathogenesis of most of these diseases entails cellmediated autoimmune inflammatory pathways, and their association with AA is well established [2]. In the same manner, the expression of CD70 was increased in human contact dermatitis, which is a Th1-mediated inflammation [22].

Additionally, the present study revealed that CD27 and CD70 gene expression was significantly lower in the nonlesional areas compared to HCs. Interestingly, Abolhassani [23] reported two family members with genetic abnormalities in the CD70- CD27 signaling cascade associated with clinical features of AA, Behcet's disease, recurrent viral pneumonia, central nervous system infection, and Hodgkin lymphoma induced by Epstein-Barr virus. The patients' clinical and immunologic data revealed an abnormality in B-cell differentiation, impaired functional activity of effector T cells, and decreased antibody production, which increased vulnerability to recurrent viral illness. The authors proposed an association between CD70 deficiency and an increased risk of alopecia areata due to either recurrent uncontrolled viral infections or decreased proliferation and activity of T-regulatory cells. This study's findings are consistent with our results of decreased CD70 and CD27 gene expression in the non-lesional scalp areas of AA patients, suggesting that a deficiency of CD70 and CD27 expression may predispose to immunological dysregulation and the development of AA. After that, the recruitment of autoreactive T cells against anagen hair follicles in active AA lesions may cause local overexpression of CD70 and CD27 expressed on the infiltrating immune cells.

Notably, several in vivo studies have suggested that monoclonal antibodies targeting CD27-CD70 complex could be a potential therapeutic modality in autoimmune diseases [5]. Anti-CD70 antibodies lowered the antibody titer and decreased joint disease's severity in murine collagen-induced arthritis [9]. In addition, anti-CD70 antibodies repressed immunoglobulin secretion by $\mathrm{B}$ cells triggered by T cells isolated from SLE patients [24]. Colitis was prevented along with a decrease in colitis-associated Th1 cytokines in a mouse model using anti-CD70 antibodies [25]. Accordingly, our study findings may shed new light on targeting CD27-CD70 complex for medical treatment of AA, especially severe cases resistant to traditional medical treatment.

Indeed, the conclusions of this study should be considered against its limitations, which include small sample size and the lack of evaluation of CD27 and CD70 tissue expression in AA scalp lesions compared to non-lesional areas and HCs. Moreover, additional research investigating the molecular functions of CD27 and CD70 in AA and comparing the expression of both molecules during AA activity and after recovery and hair regrowth is needed.

\section{Conclusion}

The expression of CD27 and CD70 genes was increased in AA scalp lesions and was associated with AA severity and activity. CD27-CD70 interaction can therefore be a predictor of AA severity and activity. Furthermore, the expression of both molecules was lower in non-lesional scalp areas. Thus, a lack of CD27 and CD70 expression may predispose to immunological dysregulation and the development of AA.

\section{Abbreviations}

AA: Alopecia areata

HF: $\quad$ Hair follicle

cT: $\quad$ Cytotoxic $T$

CD: $\quad$ Cluster differentiation

Th: $\quad$ T helper

APCs: Antigen presenting cells

HCs: Healthy controls

SALT: $\quad$ Severity of Alopecia Tool

PCR: $\quad$ Polymerase chain reactions

GAPDH: Glyceraldehyde-3-phosphate dehydrogenase

PBMNCs: Peripheral blood mononuclear cells

SLE: $\quad$ Systemic lupus erythematosus.

\section{Data Availability}

The data and materials related to the present work are included within this article. 


\section{Ethical Approval}

The study was conducted in accordance with the guidelines of the Helsinki Declaration. The study had the approval of the local Institutional Review Board and the Research Ethics Committee, Faculty of Medicine, Suez Canal University, on 25 January 2021, with the approval code: 4454.

\section{Consent}

All participants filled out a written informed consent form prior to participation in the study.

\section{Conflicts of Interest}

The authors declare that there are no conflicts of interest regarding the publication of this article.

\section{Authors' Contributions}

All authors had made substantial contributions to the following: (1) the conception and design of the study, (2) acquisition of data, data analysis, and interpretation, (3) drafting the article and revising it critically, (4) final approval of the submitted version, and (5) agreement to be accountable for all aspects of the work in ensuring that questions related to the accuracy or integrity of any part of the work are appropriately investigated and resolved.

\section{Supplementary Materials}

Supplementary table 1: demographics of the study participants $(n=80)$. Supplementary table 2: relation between CD70 gene expression in AA lesions and different data of patients with AA $(n=40)$. Supplementary table 3: correlation between CD70 gene expression in AA lesions and different data of patients with AA $(n=40)$. Supplementary table 4: relation between $\mathrm{CD} 27$ gene expression in AA lesions and different data of patients with AA $(n=40)$. Supplementary table 5: correlation between $\mathrm{CD} 27$ gene expression in AA lesions and different data of patients with AA $(n=40)$. (Supplementary Materials)

\section{References}

[1] N. A. Ismail, E. A. Toraih, H. M. Ameen, A. H. A. Gomaa, and R. E. S. M. Marie, “Association of Rs231775 genetic variant of cytotoxic T-lymphocyte associated protein 4 with alopecia areata disease in males: a case-control study," Immunological Investigations, vol. 50, no. 8, pp. 977-986, 2020.

[2] A. Alkhalifah, A. Alsantali, E. Wang, K. J. McElwee, and J. Shapiro, "Alopecia areata update," Journal of the American Academy of Dermatology, vol. 62, no. 2, pp. 177-188, 2010.

[3] R. E. S. M. Marie, N. M. Abd El- Fadeal, and L. M. Atef, "Expression of survivin and p53 genes in patients with alopecia areata: a case-control study," Australasian Journal of Dermatology, vol. 62, no. 1, pp. e29-e34, 2021.

[4] Y. Xiao, J. Hendriks, P. Langerak, H. Jacobs, and J. Borst, "CD27 is acquired by primed B cells at the centroblast stage and promotes germinal center formation," The Journal of Immunology, vol. 172, no. 12, pp. 7432-7441, 2004.
[5] B. K. Han, N. J. Olsen, and A. Bottaro, "The CD27-CD70 pathway and pathogenesis of autoimmune disease," Seminars in Arthritis and Rheumatism, vol. 45, no. 4, pp. 496-501, 2016.

[6] V. Peperzak, E. A. M. Veraar, Y. Xiao et al., "CD8+ T cells produce the chemokine CXCL10 in response to CD27/CD70 costimulation to promote generation of the CD8+ effector T cell pool," The Journal of Immunology, vol. 191, no. 6, pp. 3025-3036, 2013.

[7] H. Soares, H. Waechter, N. Glaichenhaus et al., "A subset of dendritic cells induces CD4+ T cells to produce IFN- $\gamma$ by an IL-12independent but CD70-dependent mechanism in vivo," Journal of Experimental Medicine, vol. 204, no. 5, pp. 1095-1106, 2007.

[8] A. M. Keller, A. Schildknecht, Y. Xiao, M. van den Broek, and J. Borst, "Expression of costimulatory ligand CD70 on steadystate dendritic cells breaks CD8+ T cell tolerance and permits effective immunity," Immunity, vol. 29, no. 6, pp. 934-946, 2008.

[9] E. Oflazoglu, T. E. Boursalian, W. Zeng et al., "Blocking of CD27-CD70 pathway by anti-CD70 antibody ameliorates joint disease in murine collagen-induced arthritis," The Journal of Immunology, vol. 183, no. 6, pp. 3770-3777, 2009.

[10] E. A. Olsen, M. K. Hordinsky, V. H. Price et al., "Alopecia areata investigational assessment guidelines-Part II," Journal of the American Academy of Dermatology, vol. 51, no. 3, pp. 440-447, 2004.

[11] L. C. Strazzulla, E. H. C. Wang, L. Avila et al., "Alopecia areata," Journal of the American Academy of Dermatology, vol. 78, no. 1, pp. 1-12, 2018.

[12] D. Brugnoni, P. Airò, R. Marino, L. D. Notarangelo, R. W. van Lier, and R. Cattaneo, "CD70 expression on T-cell subpopulations: study of normal individuals and patients with chronic immune activation," Immunology Letters, vol. 55, no. 2, pp. 99-104, 1997.

[13] W.-W. Lee, Z.-Z. Yang, G. Li, C. M. Weyand, and J. J. Goronzy, "Unchecked CD70 expression on T cells lowers threshold for T cell activation in rheumatoid arthritis," The Journal of Immunology, vol. 179, no. 4, pp. 2609-2615, 2007.

[14] B. K. Han, A. M. White, K. H. Dao, D. R. Karp, E. K. Wakeland, and L. S. Davis, "Increased prevalence of activated CD70+CD4+ Tcells in the periphery of patients with systemic lupus erythematosus," Lupus, vol. 14, no. 8, pp. 598-606, 2005.

[15] H. Jiang, R. Xiao, X. Lian et al., "Demethylation of TNFSF7 contributes to CD70 overexpression in CD4+ T cells from patients with systemic sclerosis," Clinical Immunology, vol. 143, no. 1, pp. 39-44, 2012.

[16] H. Yin, M. Zhao, X. Wu et al., "Hypomethylation and overexpression of CD70 (TNFSF7) in CD4+ T cells of patients with primary Sjögren's syndrome," Journal of Dermatological Science, vol. 59, no. 3, pp. 198-203, 2010.

[17] R. Speeckaert, J. Lambert, and N. van Geel, "Clinical significance of serum soluble CD molecules to assess disease activity in vitiligo," JAMA Dermatology, vol. 152, no. 11, pp. 1194-1200, 2016.

[18] M. I. El-Ghareeb, S. ElMokadem, B. Elsayed, and N. Khalifa, "Soluble CD27 and MIF as possible serum biomarkers of vitiligo activity in Egyptian patients in Sharkia Governorate," Dermatology Reports, vol. 11, no. 2, Article ID 8265, 2019.

[19] M. A. De Rie, I. M. Zonneveld, and L. Witkamp, "Soluble interleukin-2 receptor (sIL-2R) is a marker of disease activity in psoriasis: a comparison of sIL-2R, sCD27, sCD4, sCD8 and sICAM-1," Acta Dermato-Venereologica, vol. 76, no. 5, pp. 357-360, 1997. 
[20] Y. Oshikawa, T. Makino, M. Nakayama et al., "Increased CD27 expression in the skins and sera of patients with systemic sclerosis," Intractable \& Rare Diseases Research, vol. 9, no. 2, pp. 99-103, 2020.

[21] A. M. Jacobi, M. Odendahl, K. Reiter et al., "Correlation between circulating CD27highplasma cells and disease activity in patients with systemic lupus erythematosus," Arthritis \& Rheumatism, vol. 48, no. 5, pp. 1332-1342, 2003.

[22] F. Martiniuk, D. S. Lee, A. Gaspari et al., "Expression of CD70 and the TH17 transcription factor RORgammaT in human contact dermatitis," Journal of Drugs in Dermatology, vol. 7, no. 10, pp. 956-960, 2008, PMID: 19112760.

[23] H. Abolhassani, "Specific immune response and cytokine production in CD70 deficiency," Frontiers in Pediatrics, vol. 9, Article ID 615724, 2021.

[24] K. Oelke, Q. Lu, D. Richardson et al., "Overexpression of CD70 and overstimulation of IgG synthesis by lupus T cells and T cells treated with DNA methylation inhibitors," $\mathrm{Ar}$ thritis \& Rheumatism, vol. 50, no. 6, pp. 1850-1860, 2004.

[25] M. Manocha, R. Svend, A. Laouar et al., "Blocking CD27CD70 costimulatory pathway suppresses experimental colitis," The Journal of Immunology, vol. 183, no. 1, pp. 270-276, 2009. 